\section{SOIL SCIENCE}

\section{Location of Water-Table Level}

WITH the increasing use of irrigation in horticulturo and agriculture and the developing interest in the effects of evapotranspiration, it is frequently necessary to locate the level of a shallow water-table. This can be done inexpensively and effectively, using a smalldiameter observation well and an electric probe.

The observation well is constructed by inserting into an auger hole (2-in. diam.) a zinc mesh tube bound closely with strips of hessian. Zinc is preferable because it does not easily corrode, the mesh allows free movement of water through the wall of the well at any level, and the hessian acts as a filter, preventing the inwashing of fine sand and silt particles which can otherwise rapidly reduce the effective depth of the well. In order to prevent the inflow of surface water, the upper 3 in. of the well casing should be unperforated, and this section should project slightly above ground-level. Finally, precipitation can be excluded by corking or covering the open end of the well.

In an observation well of this size an ordinary dipstick would cause a considerable displacement of water. The level of the water-table can, however, be located by using a simple electric probe, similar to that described by Russell ${ }^{1}$, whose circuit is completed by contact with the water surface. Such an instrument, incorporating a $4 \cdot 5 \cdot \mathrm{V}$. dry battery and a galvanometer, can be easily constructed and obviates the necessity of using more expensive manufactured equipment. Basically, the probe consists of a stainless metal shaft which is graduated in fractions of an inch. When two contacts at the tip are closed by the water-surface the galvanometer needle is deflected and the depth can be read off against the lip of the well casing.

Twelve observation wells and a probe, constructed to the specifications described here, have been satisfactorily used by me, in soils ranging from fine clay to coarse gravel, for a period of eighteen months, in connexion with investigations of the water-table in the Thames flood-plain. It is almost certain, however, that, if necessary, these instruments would give trouble-free service for a much longer period.

Department of Geography, R. C. WARD University of Hull.

'Russell, M. B., J. Amer. Soc. Agron., 37, 408 (1945).

\section{PSYCHOLOGY}

\section{Effect of Age on Responses to Dichotic Stimulation}

The responses made by young normal adults to various methods of dichotic stimulation have been investigated and reported by Broadbent'. His observations have led him to postulate that both perceptual and storage mechanisms are necessary for the correct sequential recall of such simultaneous stimulation.

I have suggested that the defect of acquisition which has been found in elderly psychiatric patients suffering from memory disorder might be based on a breakdown of the storage mechanism postulated by Broadbent. Such patients should therefore show disturbance of recall in those half-sets of digits reproduced second in the dichotic stimulation situation, relative to the performance of a matched group of patients without memory disorder. Investigations by Caird and Inglis ${ }^{2}$ and Inglis and Sanderson ${ }^{3}$ show that this appears to be the case. Since increasing age in normal individuals also seems to affect learning capacity and as such impairment may also depend on changes in some short-term storage process it was anticipated that responses to dichotic stimulation might also show interesting associations with age.

Broadbent ${ }^{1}$ has suggested that the first half-set of digits recalled from each dichotic span does not involve the storage process. If age primarily affects storage then the reproduction of the first half-set recalled should not be affected by advancing years. If the second half-set recalled must pass through the storage process then the recall of these digits should be affected by age.

Results obtained from a preliminary analysis of the performance of 120 subjects between the ages of 11 and 70 seem to support these expectations. As age increases there is no significant impairment in the ability to recall the first half-spans. Progressive, and significantly greater, difficulty is, however, shown in the reproduction of the second half-spans. Furthermore, the longer the span to be recalled the greater the difference, overall, between the first and second halfspans $(P<0.001)$. It has never before been con. vincingly demonstrated that short-term storage ability continuously decreases with age. The work described here will be replicated before a full report is published. If these results are confirmed we shall have come to a closer specification of a fundamental process the diminished efficiency of which may produce some of the impairments of age and the breakdown of which may underly the psychosis of senility.

This work was supported by a Federal-Provincial Mental Health grant (project No. 605-5-285). The testing was carried out by Mr. W. K. Caird.

Department of Psychiatry,

JAMES INGLIS

Queen's University, Kingston, Ontario.

${ }^{1}$ Broadbent, D. E., Perception and Communication (Pergamon Press, London, 1958).

${ }^{2}$ Caird, W. K., and Inglis, J., J. Ment. Sci., 107, 1062 (1961).

${ }^{3}$ Inglis, J., and Sanderson, R. E., J. Abnorm. Soc. Psychol., 62, 709 (1961).

\section{Transfer between the Hands of a Mirror- Image Tactile Shape Discrimination}

A shape like that of the figure $C$ is seen as a $C$ in both the right and left halves of the visual field by adi lts. It is not seen as a $C$ in, say, the right and as a $D$ in the left half field. One may ask whether such an identity of the perceived shape in the two half fields is given by the structural organization of the sensory system, or whether it is the result of some learning process. To answer this question would require subjects without experience of shape and object discriminations, and yet able to perform such discriminations. Monkeys were selected as experimental subjects, and tactile discriminations were used in order to minimize the effect of previous experience with shape discriminations. In brief, the animals were trained to discriminate by touch with the left hand between the solid shapes $C$ and $D$; they were afterwards tested for their preference between the same two shapes with the right hand.

Four immature rhesus monkeys were subjects. All had been housed in the laboratory for eight months, and had been trained to perform five visual discrimin. 